\title{
E-learning package on the Mental Capacity Act 2005 for junior doctors and medical students
}

\author{
Zena Schofield, ${ }^{1}$ Ben di Mambro, ${ }^{1}$ Chris Schofield ${ }^{1}$
}

The Psychiatrist (2012), 36, 434-436, doi: 10.1192/pb.bp.112.039081

${ }^{1}$ Nottinghamshire Healthcare NHS Foundation Trust, Nottingham Correspondence to Zena Schofield (zenaschofield@doctors.org.uk)

First received 27 Feb 2012, final revision 21 May 2012, accepted 13 Jun 2012

\begin{abstract}
Aims and method The assessment of capacity is integral to all areas of medical practice; however, research has shown that doctors in many specialties have inadequate knowledge about both the Mental Capacity Act and the Mental Health Act. In this study, 39 trainee doctors and 49 fourth-year medical students completed an e-learning package, which included a pre- and post-evaluation of knowledge of the Mental Capacity Act and confidence in the use of this act.
\end{abstract}

Results In comparison to pre-intervention scores, trainee doctors and students displayed a statistically significant improvement in knowledge and subjective confidence.

\begin{abstract}
Clinical implications The e-learning package is an effective educational tool for improving knowledge about the Mental Capacity Act for trainee doctors and could be included as part of induction training for all foundation doctors as well as being considered for use in the induction programme of other trainee doctors. It is an effective tool for educating clinical medical students about the Mental Capacity Act.
\end{abstract}

Declaration of interest None.
The Mental Capacity Act 2005 was enacted in April 2007. General Medical Council (GMC) guidelines on good medical practice state that all doctors should be able to assess capacity in their patients. ${ }^{1}$ In order to assess capacity appropriately doctors need to have knowledge and understanding of the Act, however research has shown that this knowledge is lacking in doctors of all grades. ${ }^{2}$ To ensure that new doctors are competent in this area, the GMC introduced it as one of the competencies expected of new graduate doctors, ${ }^{3}$ although teaching about the Act varies from institution to institution. It has been suggested that training in mental health legislation should be mandatory for every doctor regardless of specialty. ${ }^{4}$ Consequently, as research has demonstrated, there is a need to improve knowledge in this area and an e-learning package was developed for use by foundation year 1 (F1) and foundation year 2 (F2) doctors, and individuals on general practitioner vocational training schemes (GP VTSs) and core trainees (CTl-3). This e-learning package has been piloted with doctors starting work in August 2011 as F1s at Queens Medical Centre, Nottingham, and trainees in Nottinghamshire Healthcare NHS Foundation Trust. It has also been piloted with fourth-year medical students at the University of Nottingham as they will need to be competent in assessing capacity when they start work as Fls.

Research has demonstrated that well-designed e-learning packages can improve learners' knowledge and confidence, and is comparable with traditional teaching methods. ${ }^{5}$ It has been stated that e-learning is an acceptable and effective teaching method within the medical education community. ${ }^{6}$ In addition, it has been demonstrated that e-learning for foundation doctors is equally effective as a lecture-based course for teaching about evidence-based medicine, ${ }^{7}$ suggesting that e-learning may be a suitable teaching method for doctors at this stage in their careers. It has also been suggested that well-designed e-learning packages can be useful in continuing education within psychiatry. ${ }^{8}$ Consequently, an e-learning package about the Mental Health Act for use at the beginning of foundation training posts or new posts in psychiatry was produced.

\section{Method}

We produced a web-based learning package on the Mental Capacity Act that takes approximately $20 \mathrm{~min}$ to complete. The material contained a number of case vignettes of different areas of clinical practice to allow individuals to apply knowledge during their learning. The clinical vignettes included scenarios from a variety of medical specialties including emergency medicine, orthopaedics, endocrinology, general medicine and psychiatry. Contained within the package were assessments before and after the teaching content to assess their knowledge of the Mental Capacity Act using 14 questions with true/false answers. Additionally, there were subjective rating scales of confidence of knowledge and confidence in the use of the Act pre- and post-teaching. The assessment of knowledge of the Mental Capacity Act used previously published questions used to assess knowledge of the Act among all 
grades of physicians and psychiatrists. ${ }^{2}$ Foundation year 1 doctors at Queens Medical Centre and F1s, F2s, junior doctors on the GP VTS and core trainees in psychiatry at Nottinghamshire Healthcare NHS Foundation Trust were asked to complete the package voluntarily. The e-learning package is available from the authors on request. An example of one of the vignettes in the package is shown in Box 1.

Data were collected on the pre- and post-teaching test scores to assess whether the participants' knowledge and subjective confidence in their knowledge of the Mental Capacity Act had changed. The data were collected anonymously, with only the grade of the doctor recorded for each set of data. Correct answers were not given until after the post-teaching test had been completed. In total 43 trainee doctors completed the package, of which 4 were excluded as they had not submitted information on their grade. In addition, fourth-year clinical medical students in their psychiatry module at the University of Nottingham were advised by their clinical lecturers to complete the package. In total 66 students used the package, however 17 were excluded as they had not completed both the pre- and post-package assessments, leaving results for 49 students.

\section{Results}

A total of 39 junior doctors and 49 fourth-year medical students completed both the pre- and post-teaching assessments (Table 1). Of 49 medical students, 20 got all 14 answers correct in post-assessment (41\%). Of 25 foundation trainees, 1 got all the answers correct in postassessment (4\%). Of the 14 individuals either on the GP VTS or who were core trainees, 3 got all the answers correct post-assessment (21\%). Tables 2 and 3 provide details of the mean scores for subjective confidence in knowledge and use of the Mental Capacity Act respectively.

\section{Discussion}

This study showed statistically significant improvements in knowledge about the Mental Capacity Act with use of this e-learning package for foundation and GP VTS trainees but not psychiatric core trainees, possibly owing to the small sample size of the latter group. Also there was improvement in subjective confidence in the knowledge and application of the Act, which was statistically significant. Although it is encouraging that subjective confidence levels have increased, it needs to be noted that the statistically

\section{Box 1 Example vignette}

A cyclist aged about 30 has been hit by a car and has been brought to ED [the emergency department]. He is intubated, has sustained head injuries (GCS [Glasgow Coma Scale] = 8/15), pelvic fracture with positive FAST [focused assessment with sonography for trauma] scan, haemothorax on CXR [chest x-ray], low blood pressure, and requires a blood transfusion to resuscitate him and prevent death. He also needs to go to theatre. No identification has been found on him. A man accompanied your patient in the ambulance and told the paramedic his friend (your patient) is a Jehovah's Witness and would not want a blood transfusion even if it means he will die. The friend has no written documentation to confirm or refute this information.

Would you give this patient a blood transfusion?

Answer: You would give the blood transfusion as there is no written advanced directive signed and witnessed clearly stating this man does not want a blood transfusion even if he dies. If you do not give the transfusion immediately he will die, and there is no alternative.

What authority are you using to give this blood transfusion? Answer: Mental Capacity Act - it is in the patient's best interests to keep him alive. As the outcome if left untreated would be death, then it would be proportionate to give the transfusion and proceed to theatre.

\section{Why can't you follow the friend's request?}

Options for answers:

He is not a relative. (Wrong)

The man can't prove he knows the patient. (Wrong)

You have a legal responsibility to act in his best interests and keep him alive. (Correct)

There is no written advanced directive refusing the blood transfusion. (Correct)

significant improvement in knowledge represents a relatively small increase in percentage improvement in knowledge. Despite the statistically significant improvement, it is hard to quantify the clinical significance of the improvement.

Numbers in this study were limited (probably due to it being a voluntary part of induction rather than a compulsory part), so in order to establish the generalisability of the package it would be useful to repeat it with a larger number of trainee doctors and clinical students. However, from our results it appears the package was particularly useful for less experienced non-psychiatric trainees, particularly foundation trainees who were the intended target group. The voluntary nature may also have resulted in a selection bias of either those already interested in mental health law

\begin{tabular}{|c|c|c|c|c|c|}
\hline \multirow[b]{2}{*}{ Grade } & \multirow[b]{2}{*}{$n$} & \multicolumn{2}{|c|}{ Correct answers, $n / N(\%)$} & \multicolumn{2}{|c|}{ Test statistics } \\
\hline & & Before package & After package & $\chi^{2}$ & $P$ \\
\hline Medical students & 49 & $536 / 686(78)$ & $624(91)$ & 43.2 & $<0.0001$ \\
\hline \multicolumn{6}{|l|}{ Junior doctors } \\
\hline $\mathrm{F} 1$ and $\mathrm{F} 2$ & 25 & 242/350 (69) & $281(80)$ & 11.5 & 0.0007 \\
\hline On GP VTS & 6 & $62 / 84(74)$ & $73(87)$ & 4.56 & 0.03 \\
\hline CT1-3 & 8 & $90 / 112(80)$ & $91(81)$ & 0.03 & 0.87 \\
\hline
\end{tabular}

F1, foundation year 1; F2, foundation year 2; GP VTS, general practitioner vocational training scheme; CT1-3, core trainees 1-3. 
Table 2 Mean scores of subjective confidence in knowledge of the Mental Capacity Act (MCA) ${ }^{a}$

\begin{tabular}{|c|c|c|c|c|c|}
\hline \multirow[b]{2}{*}{ Grade } & \multirow[b]{2}{*}{$n$} & \multicolumn{2}{|c|}{ Mean score of confidence in knowledge of the MCA } & \multicolumn{2}{|c|}{ Wilcoxon signed rank } \\
\hline & & Before package & After package & $w$ & $P$ \\
\hline Medical students & 49 & 3.2 & 4.2 & -496 & $<0.0001$ \\
\hline \multicolumn{6}{|l|}{ Junior doctors } \\
\hline $\mathrm{F} 1$ and $\mathrm{F} 2$ & 25 & 2.4 & 3.6 & -200 & 0.0001 \\
\hline On GP VTS and CT1-3 & 14 & 3.0 & 3.9 & -45 & 0.005 \\
\hline
\end{tabular}

F1, foundation year 1; F2, foundation year 2; GP VTS, general practitioner vocational training scheme; CT1-3, core trainees 1-3.

a. On a Likert scale range $1-5$ (1, poor; 3 , neutral; 5 , excellent).

\begin{tabular}{|c|c|c|c|c|c|}
\hline \multirow[b]{2}{*}{ Grade } & \multirow[b]{2}{*}{$n$} & \multicolumn{2}{|c|}{ Mean score of confidence in applying the MCA } & \multicolumn{2}{|c|}{ Wilcoxon signed rank } \\
\hline & & Before package & After package & $w$ & $P$ \\
\hline Medical students & 49 & 2.9 & 3.9 & -542 & $<0.0001$ \\
\hline \multicolumn{6}{|l|}{ Junior doctors } \\
\hline $\mathrm{F} 1$ and $\mathrm{F} 2$ & 25 & 2.1 & 3.5 & -201 & 0.0001 \\
\hline On GP VTS and CT1-3 & 14 & 3.0 & 3.7 & -28 & 0.001 \\
\hline
\end{tabular}

F1, foundation year 1; F2, foundation year 2; GP VTS, general practitioner vocational training scheme; CT1-3, core trainees 1-3.

a. On a Likert scale range 1-5 (1, poor; 3 , neutral; 5 , excellent).

taking part or those aware of their lack of confidence in this area, or both. The pre-package results are better than in previous studies, ${ }^{2}$ suggesting baseline knowledge of the sample group was high, yet despite this there was still improvement in knowledge and subjective confidence after using the package. The study has only considered the benefit of an e-learning package. Further research could be undertaken to compare the e-learning package with an alternative educational method.

\section{Implications}

Despite a modest sample size there are statistically significant improvements in the knowledge about the Mental Capacity Act in trainee doctors and clinical students and their subjective confidence in their knowledge and use of the Act following using the e-learning package. Consequently, it would be reasonable to suggest considering using the package as part of induction for foundation trainee doctors of any specialty. Although the tool is an effective educational tool for improving knowledge and subjective confidence, it would still be vital for all trainee doctors to receive adequate clinical supervision in this area of their clinical practice, as in all areas of clinical practice.

\section{About the authors}

Zena Schofield is a specialty registrar in adult psychiatry in the Nottinghamshire Healthcare NHS Foundation Trust. Ben di Mambro is consultant perinatal psychiatrist and clinical teaching fellow in the Nottinghamshire Healthcare NHS Foundation Trust. Chris Schofield is consultant liaison psychiatrist in the Nottinghamshire Healthcare NHS Foundation Trust and an approved clinician regional training lead, a Section 12 approval training lead and Deprivation of Liberty Safeguards trainer for the Royal College of Psychiatrists' Education and Training Centre.

\section{References}

1 General Medical Council. Consent: Patients and Doctors Making Decisions Together. GMC, 2008.

2 Schofield C. Mental Capacity Act 2005 - what do doctors know? Med Sci Law 2008; 48: 113-6.

3 General Medical Council. Outcome 2: the doctor as a practitioner. In Tomorrow's Doctors: para 13. GMC, 2009.

4 Schofield C. Mental health law training should be mandatory for all doctors. Commentary on ... Knowledge of mental health legislation in junior doctors training in psychiatry. Psychiatrist 2011; 35: 466-8.

5 Chumley-Jones $\mathrm{H}$, Dobbie A, Alford CL. Web-based learning: sound educational method or hype? A review of the evaluation literature. Acad Med 2002; 77: s86-93.

6 Ruiz J, Mintzer MJ, Leipzig RM. The impact of e-learning in medical education. Acad Med 2006; 81: 207-12.

7 Hadley J, Kulier R, Zamora J, Coppus SF, Weinbrenner S, Meyerrose B, et al. Effectiveness of an e-learning course in evidence-based medicine for foundation (internship) training. J R Soc Med 2010; 103: 288-94.

8 Hare EE. E-learning for psychiatrists. Psychiatrist 2009; 33: 81-3. 